\title{
Monitoring Coastal Sea Level Using Reflected GNSS Signals
}

\author{
Johan S. Löfgren, Rüdiger Haas, Jan M. Johansson \\ Chalmers University of Technology, Department of Earth and Space Sciences, \\ Onsala Space Observatory, SE-439 92 Onsala (Sweden)
}

Abstract

A continuous monitoring of coastal sea level changes is important for human society since it is predicted that up to 332 million people in coastal and low-lying areas will be directly affected by flooding from sea level rise by the end of the 21st century. The traditional way to observe sea level is using tide gauges that give measurements relative to the Earth's crust. However, in order to improve the understanding of the sea level change processes it is necessary to separate the measurements into land surface height changes and sea surface height changes. These measurements should then be relative to a global reference frame. This can be done with satellite techniques, and thus a GNSS-based tide gauge is proposed. The GNSS-based tide gauge makes use of both GNSS signals that are directly received and GNSS signals that are reflected from the sea surface. An experimental installation at the Onsala Space Observatory (OSO) shows that the reflected GNSS signals have only about $3 \mathrm{~dB}$ less signal-to-noise-ratio than the directly received GNSS signals. Furthermore, a comparison of local sea level observations from the GNSS-based tide gauge with two stílling well gauges, located approximately $18 \mathrm{~km}$ and $33 \mathrm{~km}$ away from OSO, gives a pairwise root-mean-square agreement on the order of $4 \mathrm{~cm}$. This indicates that the GNSS-based tide gauge gives valuable results for sea level monitoring.

Key words: GNSS, reflected signals, local sea level monitoring, tide gauge

\section{Introduction}

Global climate change is resulting in the melting of large masses of ice in polar and subpolar regions, bringing freshwater into the ocean, and chang-

Email addresses: johan.lofgren@chalmers.se, rudiger.haas@chalmers.se, jan.johansson@chalmers.se (Johan S. Löfgren, Rüdiger Haas, Jan M. Johansson). 
ing the sea level (Bindoff et al., 2007). Recent results from, e.g., ice melt in Antarctica and Greenland even show accelerated melting (Velicogna, 2009) and in addition, other effects from global warming, e.g., thermal expansion of sea water and changes in atmospheric and ocean circulation will further impact the sea level. This will have important consequences for human society. In Watkins et al. (2007) it is predicted that up to 332 million people in coastal and low-lying areas will be directly affected by flooding from sea level rise by the end of the 21st century. Furthermore, the displacement of these people will affect millions more. This danger for human society requires therefore a continuous monitoring of the sea level.

The traditional way to measure the sea level, by tide gauges, is to measure the vertical distance between the sea surface and the land surface, which is directly related to the volume of the ocean. This observation, called local sea level, results in measurements that are relative to the Earth's crust (Scherneck et al., 2002). However, in order to fully understand the underlying processes, a separation into local land surface height changes and sea surface height changes is necessary. This can be done by measuring them both separately and relative to a more appropriate global reference frame which, e.g., has its origin in the Earth's center of mass.

Satellite techniques, e.g., Global Navigation Satellite Systems (GNSS), can be used to determine the motion of the Earth's crust in relation to the center of mass (Johansson et al., 2002). By observing directly received and reflected GNSS signals from the sea surface, information of both sea surface height changes and land surface height changes can be obtained. Furthermore, combining both measurements results in the quantity local sea level change. Therefore, a GNSS-based tide gauge is proposed.

\section{Concept of the GNSS-based tide gauge}

The proposed GNSS-based tide gauge consists of two antennas, one zenithlooking right hand circular polarized (RHCP) and one nadir-looking left hand circular polarized (LHCP), mounted back-to-back on a beam over the ocean (see Fig. 1). The idea is to mount the antennas so that the phase centers are aligned along the local vertical, thus there is no horizontal distance between the positions of the phase centers and they differ only in vertical position.

The concept builds upon two standard geodetic-type two-frequency GNSS receivers that are connected to these two antennas (one to the RHCP and one to the LHCP antenna). Thus, there is no need for special customized GNSS receivers as, e.g., described by Belmonte Rivas \& Martin-Neira (2006). Neither does the concept include an interferometric approach as, e.g., presented by 
Rius et al. (2009). Instead, the carrier phase delay data from the two receivers are analyzed using relative positioning (see Sec. 5) as applied in standard geodetic analysis, similar to Martin-Neira et al. (2002).

The RHCP antenna receives the direct GNSS signals, whereas the LHCP antenna receives the signals that are reflected from the sea surface. When the signals are reflected, they change polarization from RHCP to LHCP. The reflected signals experience an additional path delay, as compared to the directly received signals. This means that the LHCP antenna can be regarded as a virtual RHCP antenna located below the sea surface, and this virtual antenna is depicted in blue in Fig. 1. The height of the LHCP antenna over the sea surface $(h)$ corresponds to

$$
h=\frac{(a+b) / \sin \varepsilon-d}{2}
$$

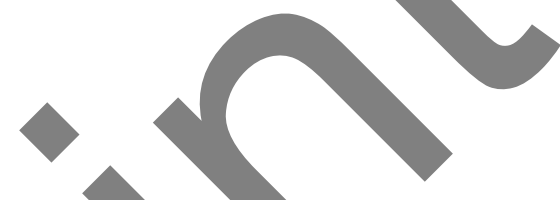

where $a+b=c$ is the additional path delay of the reflected signal, $\varepsilon$ is the elevation of the transmitting satellite, and $d$ is the vertical separation between the phase centers of the RHCP and the LHCP antennas. When the sea level changes, the path delay of the reflected signal changes. Thus, the LHCP antenna will appear to change vertical position. The vertical difference in position between the RHCP and the LHCP antenna $(\Delta v)$ can from the geometry in Fig. 1 easily be related to the height of the LHCP antenna over the sea surface according to

$$
\Delta v=2 h+d
$$

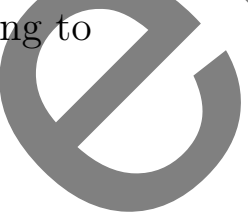

Since the height of the LHCP antenna over the sea surface is directly proportional to the sea surface height and the RHCP antenna is directly proportional to the land surface height, the installation monitors changes in local sea level. Moreover, according to Eq. 2 a change in sea level is detected by a change in the vertical position between the antennas, which is twice as big as the change in sea level.

In Fig. 1 it is assumed that the sea surface is flat and that the reflection is specular, meaning that all of the signal energy is reflected coherently towards the antenna. However, in the real case, the distribution of the reflected energy is governed by the surface roughness compared to the signal wavelength and elevation angle (Rees, 2003), e.g., a high surface roughness will spread the reflected signal more in space than a low surface roughness. The effect for the GNSS-based tide gauge will be that for large sea surface roughness, the GNSS receivers will have difficulties to keep lock on the satellites. A first attempt to reduce this could be to place the antenna over a naturally calm water surface, i.e., in a bay or in the vicinity of islands that can shelter from winds and ocean swell. 
Multiple satellites with different elevation and azimuth angles are observed each epoch and will give rise to reflected signals with different incidence angles from different directions. This means that the estimated change in sea level can not be considered to originate from one specific point on the sea surface, but rather represents the change of an average sea surface formed by the reflection points. The distribution of these points is limited by the placement of the antenna (height of the antenna, the landmass on which the antenna is positioned, and also obstacles in the water) and the antenna geometry. As an example, the expected reflection points on the sea surface from the GPSsignals are presented in Fig. 2 for the antenna height of $1 \mathrm{~m}$ over the sea surface. The reflections are shown for 24 hours with an eleyation cut-off of $15^{\circ}$, limiting the extension of the reflective surface. For an antenna that is placed higher above the reflective surface, the reflection points will have the same spatial distribution as in Fig. 2, but with a larger scale (larger radius).

In Fig. 3 the maximum area of the reflection points between $90^{\circ}$ and $270^{\circ}$ azimuth (half circle), is presented for different heights of the antenna. It can be seen that an antenna placed higher corresponds to a larger reflective surface. However, by increasing the height of the antenna, while the sampling frequency is fixed, the spatial resolution of the surface will decrease. Furthermore, the antenna geometry will obscure reflected signals from higher satellite elevations (reflection points close to the antenna). This effect decreases when increasing the height of the antenna.

Additionally, the antenna height range in Fig. 3 can also be viewed as tidal range. When the tide is low, the height of the antenna over the sea surface is high corresponding to a large reflective surface, whereas when the tide is high, the height of the antenna is low corresponding to a small reflective surface. This means that the spatial sampling will be different during different stages of the tidal cycle.

\section{$3 \quad$ Experimental setup}

An experimental setup of the GNSS-based tide gauge was installed in December 2008 over the ocean at the Onsala Space Observatory (OSO) at the west coast of Sweden. The antenna installation was mounted on a wooden deck, built on the coastal bedrock, so that there was open sea water in a southward direction. This was done in order to maximize the potential reflective surface (at these latitudes $57^{\circ} \mathrm{N}$ the visibility of satellites to the north is limited), but also to ensure power supply and shelter for the receivers in a nearby boathouse. The downward-looking LHCP antenna was positioned approximately $1 \mathrm{~m}$ over the sea surface (at the time of installation) and both antennas were protected by hemispherical radomes, see Fig. 4. The position 
over the sea surface was chosen on the basis of preventing the installation from water damage and since the tidal range at Onsala is quite small, approximately $20 \mathrm{~cm}$, a height of $1 \mathrm{~m}$ over the surface at the time of installation was decided to be enough. In this experimental installation it was not possible to realize the goal to align the antennas in an optimal way (see Sec. 2). Instead the vertical and horizontal distances between the antenna reference points were measured and used as corrections in the data analysis.

Data were collected during three days using two Leica GRX1200 receivers (one for each antenna). The receivers were connected to one RHCP antenna (Leica AT504 GG choke-ring) for the direct signal, and one LHCP antenna (Leica AR25 multi-GNSS choke-ring) for the reflected signal. Both receivers recorded 40 hours of continuous data with $20 \mathrm{~Hz}$ sampling.

\section{Signal evaluation}

The signal-to-noise ratio (SNR) as determined by the two receivers was used as a first data quality check for the received signals. As an example, in Fig. 5a and Fig. 5b the SNR from 12 hours of observation is shown versus azimuth and elevation respectively, for the upward-looking RHCP antenna (direct signal), and in Fig. 5c and Fig. 5d for the downward-looking LHCP antenna (reflected signal). It can be seen that the receiver connected to the RHCP antenna received GNSS signals from all azimuth directions. However, it has a clear drop in SNR in the north direction between approximately $-16^{\circ}$ and $+6^{\circ}$ azimuth. The LHCP antenna received GNSS signals from all azimuth directions except the north direction between approximately $-16^{\circ}$ and $+22^{\circ}$ azimuth. Comparing the SNR of the RHCP antenna with the SNR of the nearby International GNSS Service (IGS) station ONSA (see Fig. 5e and Fig. 5f) during the same 12 hours, a similar SNR decrease is seen for northern azimuth angles. This SNR decrease is due to the geographical position of this site at the latitude of $57^{\circ}$ and the orbital inclination of the satellites.

The surrounding to the north of the GNSS-based tide gauge installation, between $-135^{\circ}$ and $+45^{\circ}$ azimuth, was dominated by bedrock from the coastline and a boathouse, while all other directions were open sea. This means that the upward-looking antenna was shielded from signals from certain northern azimuth angles, explaining the sparse SNR coverage to the north for the RHCP antenna compared to the IGS station. Additionally, comparing the location of the coastline to the azimuth angles of the reflected signals (Fig. 5c), there are reflections from the direction of the bedrock coastline indicating multipath reflections. Therefore, in order to avoid influences of multipath, we applied an azimuth mask for the north direction to exclude this region (from $-135^{\circ}$ to $+45^{\circ}$ azimuth) and the results are shown in Fig. 5g, Fig. 5h, Fig. 5i, and 
Fig. 5j for both the direct and reflected signals. The SNR of the remaining data (from the direction of the sea) resembles the IGS station SNR, meaning a significant increase in SNR above about $20^{\circ}$ elevation for both the direct and reflected signals, see Fig. 5h and Fig. 5j. Moreover, it can be seen that the reflected signals are noisier than the direct signals (especially below $20^{\circ}$ elevation). However, the SNR for the reflected signals is always above $25 \mathrm{~dB}$ and it increases with increasing elevation.

After applying the azimuth mask for the LHCP antenna there were still some SNR fluctuation for lower elevations (see Fig. 5j). The reason for this could be multipath or interference from the direct RHCP signal received through the backside of the antenna. In order to investigate the latter, an approximate antenna radiation pattern for the Leica AR25 multi-GNSS chokering antenna for GPS L1 signals (Bedford et al., 2009) is presented in Table 1. The reflected signal corresponds to the LHCP signal (RHCP reflected in the sea surface) and the direct signal corresponds to the RHCP signal. The signal strength is measured in decibel isotropic circular (dBic) and is shown for different satellite elevations. In Table 1 we see that the difference in gain decreases with elevation. However, the reflected LHCP signal (received through the antenna front side) is approximately $7 \mathrm{dBic}$ higher than the direct RHCP signal (received through the antenna backside) for all elevations. This indicates that the antenna greatly suppresses the direct RHCP signal and that the SNR fluctuations therefore should not originate from interference between the direct and reflected signals.

In order to characterize the sea state during the experiment, we used model data of significant height of wind waves and swell provided by the Swedish Meteorological and Hydrological Institute (SMHI). The data were produced from the Simulating WAves Nearshore (SWAN) model (Holthuijsen et al., 1993) with forcing from a regional climate model and are presented in Fig. 6. Here data are presented for a SWAN model grid point located at N 57.46 E 11.95, 1.e., close to the GNSS-based tide gauge which is located at N 57.39 E 11.92. In Fig. 6, we see that the significant height of wind waves and swell is below $0.2 \mathrm{~m}$ during most of the experiment. Furthermore, the placement of the GNSS-based tide gauge is quite sheltered with several smaller islands spread out in the vicinity, reducing both wind and ocean swell. Hence, the actual local values of significant height is most likely smaller. The low values of significant height of wind waves and swell during the experiment indicates that most of the signals were reflected coherently. We suspect that most of the SNR fluctuations originate from multipath from the bedrock and the installation.

In order to evaluate the average difference in SNR between the direct and the reflected signals, a second order polynomial was fitted to the SNR (versus elevation) of each antenna for the 12 hours, see Fig. 5h and Fig. 5j. The average SNR difference between the two SNR polynomials was between $1.0 \mathrm{~dB}$ and 


\section{Data analysis}

The analysis strategy was to produce hourly estimates of the vertical difference between the RHCP and LHCP antennas, and to compare the results to other independent data sets. First, to enable faster processing, the $20 \mathrm{~Hz}$ data were decimated to $1 \mathrm{~Hz}$ data using the Translation, Editing, and Quality Check (TEQC) software (Estey \& Meertens, 1999). Second, an elevation and azimuth mask was applied, removing data below $20^{\circ}$ elevation and outside the south direction $-135^{\circ}$ to $+45^{\circ}$ azimuth. This was done in order to remove unwanted signals, e.g., multipath from the coastline and to remove signals at low elevations. Reflected signals from low elevations have low SNR since they are highly elliptical polarized (at the Brewster angle, about $8^{\circ}$, even purely horizontal polarized). However, for elevation angles greater that the Brewster angle the ellipticity decreases with elevation (Hannah, 2001).

Thereafter, an in-house developed software for relative positioning in MATLAB was used to analyze the data. The software uses GPS L1 phase delays for single differences together with IGS satellite ephemerides (Dow et al., 2009) according to

$$
\lambda \Phi_{A B}^{j}(t)=\varrho_{A B}^{j}(t)+\lambda N_{A B}^{j}+c \delta_{A B}(t)
$$

where $\lambda$ is the wavelength of the L1 carrier, $\Phi_{A B}^{j}(t)$ is the difference in the measured carrier phase expressed in cycles, $\varrho_{A B}^{j}(t)$ is the difference in geometry in meters, $N_{A B}^{j}$ is the difference in phase ambiguity in cycles, $c$ is the speed of light in vacuum, and $\delta_{A B}(t)$ is the difference in receiver clock bias. $A$ and $B$ denotes the two receivers and $j$ denotes the satellite used in the single difference. Because of the short baseline between the receivers, both tropospheric and ionospheric effects were ignored in Eq. 3.

The difference in geometry can then be expressed in a local coordinate system using azimuth $\alpha$ and elevation $\varepsilon$ for each satellite as

$$
\varrho_{A B}^{j}(t)=-\Delta e \sin \left(\alpha^{j}\right) \cos \left(\varepsilon^{j}\right)-\Delta n \cos \left(\alpha^{j}\right) \cos \left(\varepsilon^{j}\right)-\Delta v \sin \left(\varepsilon^{j}\right)
$$

where $\Delta e, \Delta n$, and $\Delta v$ are the east, north, and vertical components of the baseline between the two receivers. In this case the single difference is defined as the LHCP phase minus the RHCP phase, hence the negative sign before the three components. Since, in our case, both the east and north baseline 
components were known, these two contributions were moved to the left side of Eq. 3, resulting in the following linear system of equations

$$
y=A x+\epsilon
$$

Here $y$ is the vector of observed single differenced phase measurements together with east and north baseline corrections; $A$ is the design matrix containing the partial derivatives for the vertical baseline, phase ambiguity differences for each satellite pair, and difference in receiver clock bias; $x$ contains the parameters to be estimated (vertical baseline, phase ambiguity differences, and differences in clock bias), and $\epsilon$ is the unmodeled effects and measurement noise.

Each least squares solution used 20 minutes of data (around the full hour), solving for the local vertical component for this interval, phase ambiguity differences for each satellite pair, and receiver clock differences for each epoch (every second). Solutions were made for full hours where the receivers had continuous track, meaning no cycle slips, of at least 2 satellites (usually 4 to 5 satellites) during the 20 minutes. Furthermore, satellites with continuous track for at least 10 minutes during the interval were also incorporated in the solution. This approach resulted in solutions for 27 hours.

Since the differences in local vertical coordinate corresponds to the local sea level according to Eq. 2, the vahues were converted into a time series of local sea level $h$. In this time series the values are relative to the upward-looking RHCP antenna and are therefore negative. Adding the ellipsoidal height of the RHCP antenna to these values will thus give local sea level in an ellipsoidal system. It is of course also possible to add the geoid height, which will give measurements of true sea level.

\section{Results}

The resulting time series of local sea level from the hourly solutions were compared to independent data from two stilling well gauges operated by SMHI at Ringhals and Göteborg, about $18 \mathrm{~km}$ south of and $33 \mathrm{~km}$ north of OSO, respectively (see Fig. 7). Since the local sea level observations from the GNSSbased tide gauge are relative to the position of the RHCP antenna, and the local sea level measurements from SMHI referred to the mean sea level of the year, mean values were removed from each time series. This allows to compare the three time series in a meaningful way avoiding any biases. The results are presented in Fig. 8 were the error bars in the GNSS-based tide gauge time series consists of the standard deviation from each least squares solution 
multiplied by a factor of 10 . This was done to make the error bars clearly visible.

In order to investigate the agreement of the three time series, the pairwise mean, maximum, and root-mean-square (RMS) differences were calculated. The values are given in Table 2 and show that the RMS agreement between the three independent time series is better than $4 \mathrm{~cm}$. The maximum difference for the GNSS-based tide gauge $(<9.3 \mathrm{~cm})$ is higher than the IOC (2006) accuracy requirement of $1 \mathrm{~cm}$ at all times. However, this is also true for the stilling well gauges $(7.5 \mathrm{~cm})$ and the main reason is most likely due to the different locations of the tide gauges. Overall, the stilling well gauges appear to agree slightly better with respect to each other than the GNSS-based tide gauge with each of them. This indicates that there might still be some systematic effects that need further investigation.

These effects might originate from how the two different sea level observations were obtained. The local sea level results from the stilling well gauges are interpolated values to the full hour from measurements done 5 minutes before and after each full hour (Hammarklint, 2010). These values are the mean values of 4 samples spread in time by 15 seconds. The GNSS-derived local sea level results are, on the other hand, based on analysis of 20 minutes of data around every full hour in order to fully resolve the phase ambiguity differences. Thus, the temporal averaging of the stilling well gauges and the GNSS-derived local sea level results is significantly different. A future enhancement of the GNSS-based tide gauge would therefore be to reduce the number of epochs needed in order to produce an accurate local sea level observation.

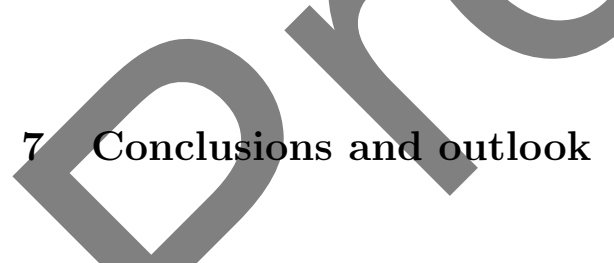

The experimental GNSS-based tide gauge installation shows that it is possible to receive GNSS signals reflected in the sea surface using standard geodetic GNSS equipment. As expected the reflected signals were noisier than the direct signals and the observed noise was larger at low elevations. The average SNR difference between the direct and the reflected signals was between $1.0 \mathrm{~dB}$ and $3.4 \mathrm{~dB}$.

The GNSS-derived time series of sea level resembles reasonably well the independently observed data from the two traditional tide gauges, and the RMS agreement is better than $4 \mathrm{~cm}$. The two traditional tide gauges agree slightly better than the GNSS-based tide gauge with each of them, indicating that there might be unaccounted systematic effects. One reason could be the different temporal averaging of the results, i.e., average values of 1 minute from the traditional tide gauges compared to average values over 20 minutes from 
the GNSS analysis. Another reason could be hydrodynamic differences due to the coastline geometry at Ringhals, OSO, and Göteborg. Additionally, antenna phase center variations could contribute to the RMS differences. The upwardlooking RHCP antenna and the downward-looking LHCP antenna were two different types of antennas and corrections for phase center variations were not applied in the data analysis.

The agreement between the measurements with the GNSS-based tide gauge and the traditional tide gauges indicates that the GNSS-based tide gauge gives valuable results for sea level monitoring. Therefore, we are currently installing the GNSS-based tide gauge permanently at OSO. For this installation we plan to exchange the upward-looking GNSS antenna to an antenna of the same type as the downward-looking one, but RHCP, in order to minimize systematic effects due to, e.g., phase center variation differences. Qur ambition is to develop strategies for a real-time sea level monitoring with this installation and also to derive tidal constituents (amplitude and phase) and compare them to theoretical models.

Additionally, we plan to install a pressure sensor based tide gauge at the same site in order to further evaluate the GNSS-based tide gauge, e.g., by comparing time series with identical temporal resolution, and to avoid any effects due to hydrodynamical differences caused by different coastal geometry.

With simulations, we have seen that the spatial sampling depends on the stage of the tidal cycle, i.e., at low tide the reflective surface is larger than at high tide. For OSO which has a quite small tidal range of approximately $20 \mathrm{~cm}$, there is not a big difference, but for a site with larger tidal (and meteorological) range this can have an impact on the measurements, especially if the temporal resolution is high. However, in our case, where we have basically averaged over 20 minutes of data to próduce one sea level observation, there should not be any significant difference.

In order to measure land surface height changes, the GNSS-based tide gauge needs to be firmly connected to the land surface. This can be monitored with point positioning of the RHCP antenna and relative positioning between the RHCP antenna and the nearby $(\sim 800 \mathrm{~m})$ IGS station ONSA. The IGS station position is well know to better than the mm-level. Therefore, a relative solution between the two antennas could reveal possible motions in the tide gauge installation.

For the future, we need to reduce the number of epochs needed in order to produce an accurate local sea level observation. The main reason for the low temporal resolution in our processing was difficulties with estimating the ambiguity parameters. There are, however, several other techniques for estimating phase ambiguities available and in the future we should be able to 
increase the temporal resolution significantly.

Furthermore, we plan to analyze the high-rate $(20 \mathrm{~Hz})$ observations in postprocessing. This high sampling rate might allow us to derive parameters that describe the sea surface roughness, as well as sea water properties.

\section{Acknowledgements}

We would like to thank Per Jarlemark at the Technical-Research Institute of Sweden (SP) for valuable insights in GPS data processing. Additionally we would like to thank the Swedish Meteorological and Hydrological Institute (SMHI) for providing us with data from the traditional tide gauges. Finally we would also like to thank Adlerbertska Forskningsstiftelsen for partially funding this project.

\section{References}

Bedford, L., Brown, N. \& Walford, J. Leica AR25 White paper. Leica Geosystems AG, Heerbrugg, Switzerland, Available at http://www.leicageosystems.com/common/shared/downloads/inc/downloader.asp?id=10904, 2009.

Belmonte Rivas, M. and Martin-Neira, M. Coherent GPS reflections from the sea surface. IEEE Geosci. Remote Sens. Lett. 3, 28-31, 2006.

Bindoff, N. L., Willebrand, J., Artale, V. et al. Observations: Oceanic climate change and sea level, in Climate Change 2007: The Physical Science Basis. Contribution of Working Group I to the Fourth Assessment Report of the Intergovernmental Panel on Climate Change, edited by S. Solomon, D. Qin, M. Manning, Z. Chen, M. Marquis, K. B. Averyt, M. Tignor, \& H. L. Miller, Cambridge University Press, Cambridge, United Kingdom and New York, NY, USA, 2007.

Dow, J. M., Neilan, R. E., \& Rizos, C. The International GNSS Service in a changing landscape of Global Navigation Satellite Systems, J. Geod. 83, 191-198, 2009.

Estey, L. H., Meertens, C. M. TEQC: The multi-purpose toolkit for GPS/GLONASS data. GPS Solutions, 3(1), 42-49, 1999.

Hammarklint, T., Swedish Meteorological and Hydrological Institute (SMHI), Norrköping, Sweden, Personal communication, 2010.

Hannah, B. M. Modelling and simulation of GPS multipath propagation. PhD thesis, Queensland University of Technology, 2001.

Holthuijsen, L. H., Booij, N., \& Ris, R. C. A spectral wave model for the 
coastal zone, Proc. of 2nd Int. Symposium on Ocean Wave Measurement and Analysis, New Orleans, USA, 630-641, 1993.

IOC Manual on sea-level measurement and interpretation, Volume 4: An update to 2006. Paris, Intergovernmental Oceanographic Commission (IOC) of UNESCO, Manuals and Guides, 14, 78 pp, 2006.

Johansson, J. M., Davis, J. L., Scherneck, H.-G. et al. Continuous GPS measurements of postglacial adjustment in Fennoscandia 1. Geodetic results. J. Geophys. Res. (Solid Earth) 107, 2157, 2002.

Martin-Neira, M., Colmenarejo, P., Ruffini, G. et al. Altimetry precision of $1 \mathrm{~cm}$ over a pond using the wide-lane carrier phase of GPS reflected signals, Can. J. Remote Sens., 28, 3, 394-403, 2002.

Rees, W. G. Physical principles of remote sensing, second edition, University press, Cambridge, United Kingdom, 2003.

Rius, A., Nogués-Correig, O., Ribó S. et al. PARIS interferometric technique: proof of concept, in: Proceedings of 2nd International Colloquium-Scientific and Fundamental Aspects of the Galileo Programme, Proceedings CD ROM, European Space Agency, 2009.

Scherneck, H.-G., Johansson, J. M., Elgered, G. et al, BIFROST: Observing the three-dimensional deformation of Fennoscandia, in. Ice Sheets, Sea Level and the Dynamic Earth, J. X. Mitrovica and B. Vermeersen (Eds). AGU Geodyn. Ser. 29, 69-93. American Geophysical Union, Washington D.C., 2002.

Velicogna, I. Increasing rates of ice mass loss from the Greenland and Antarctic ice sheets revealed by GRACE, Geophys. Res. Lett., 36, L19503, 2009.

Watkins, K., Ugaz, C., Carvajal, L. et al. Human Development Report 2007/2008 - Fighting climate change: Human solidarity in a divided world, Palgrave Macmillan, New York, USA, for United Nations Development Programme, Available at http://hdr.undp.org/en/reports/global/hdr2007-

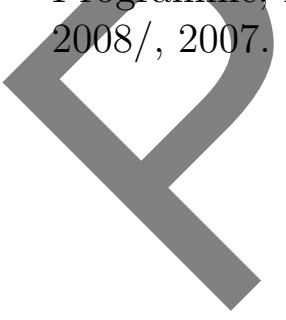


Table 1

Approximate values for antenna gain of the direct and reflected GPS L1 signals from different elevation angles for the downward looking LHCP antenna. The table is based on the antenna radiation pattern for AR25 (Bedford et al., 2009). Values are in decibel isotropic circular and are normalized so that the peak gain is $0 \mathrm{dBic}$. Reflected means LHCP signals received through the antenna front side and direct means RHCP signals received through the antenna backside.

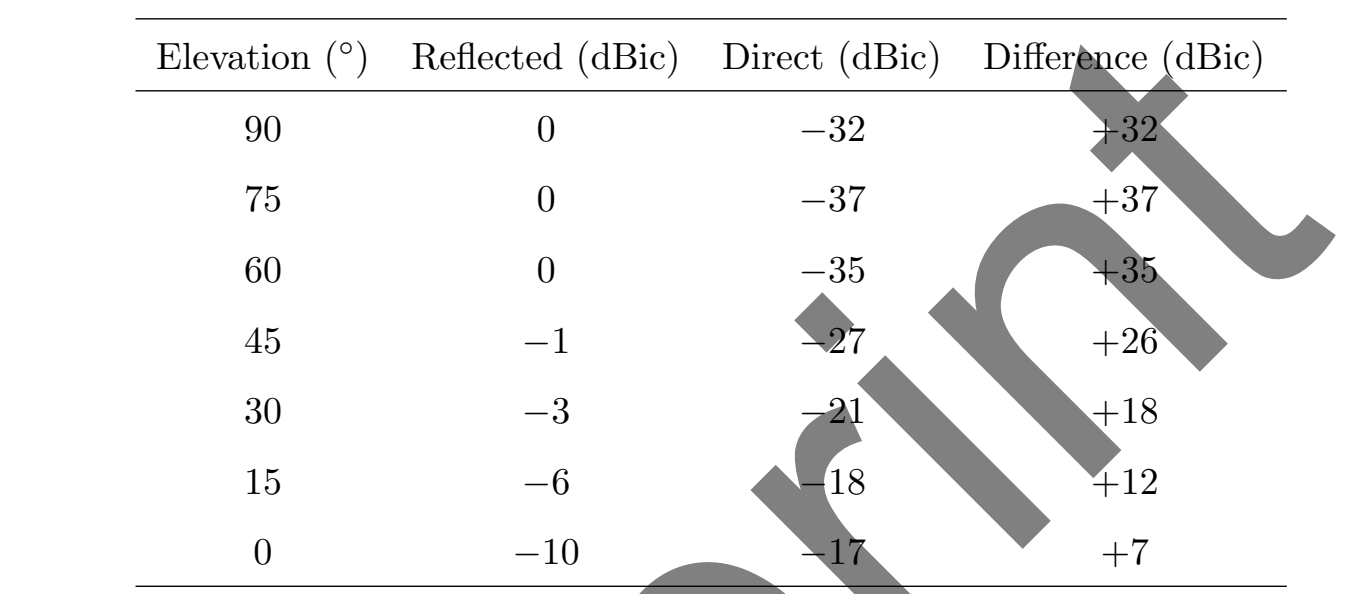

Table 2

Pairwise mean, maximum, and RMS differences in local sea level between the GNSSbased tide gauge at the Onsala Space Observatory (OSO) and the stilling well gauges at Ringhals and Göteborg.

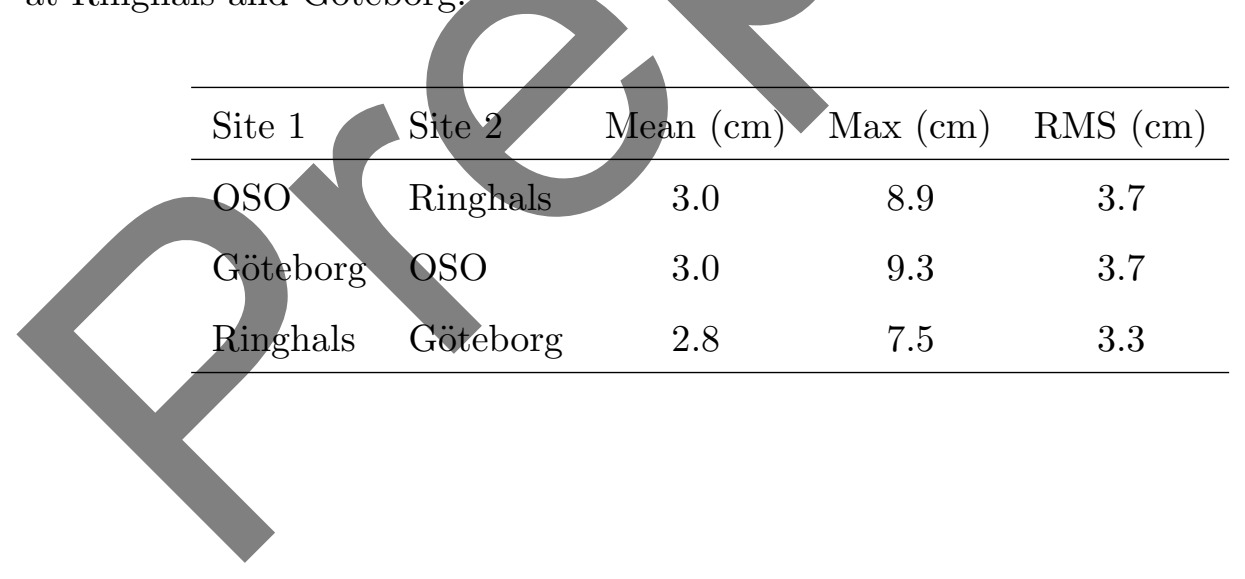




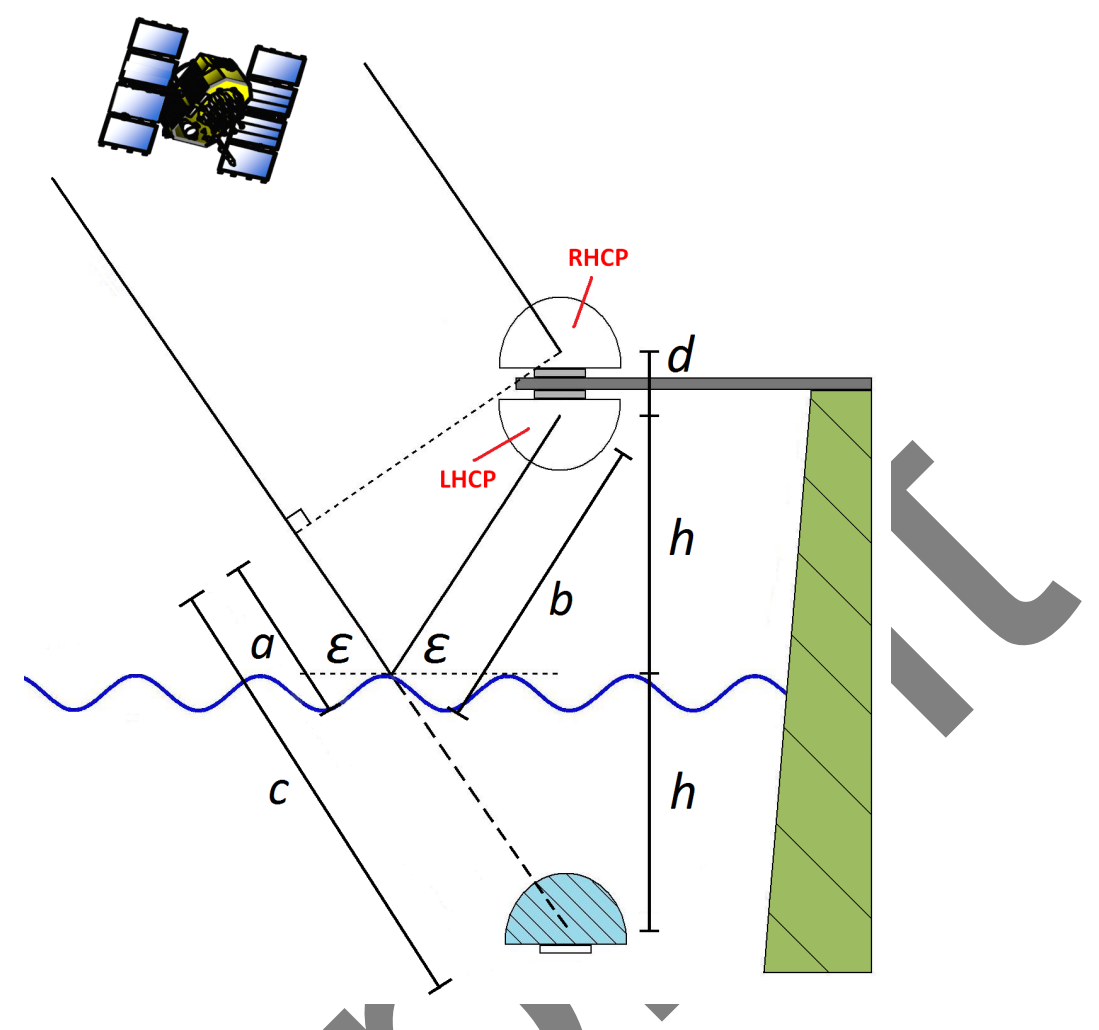

Fig. 1. Schematic drawing of the GNSS-based tide gauge. The installation consists of two antennas (one RHCP and one LHCP), separated vertically by a distance $d$, mounted on a beam over the ocean at the height $h$ over the sea surface. The LHCP antenna receives the reflected GNSS signals whereas the RHCP antenna receives the direct signals. Because of the additional path delay for the signals received with the LHCP antenna $(a+b=c)$, the antenna will appear to be positioned at the distance $h$ under the sea surface.
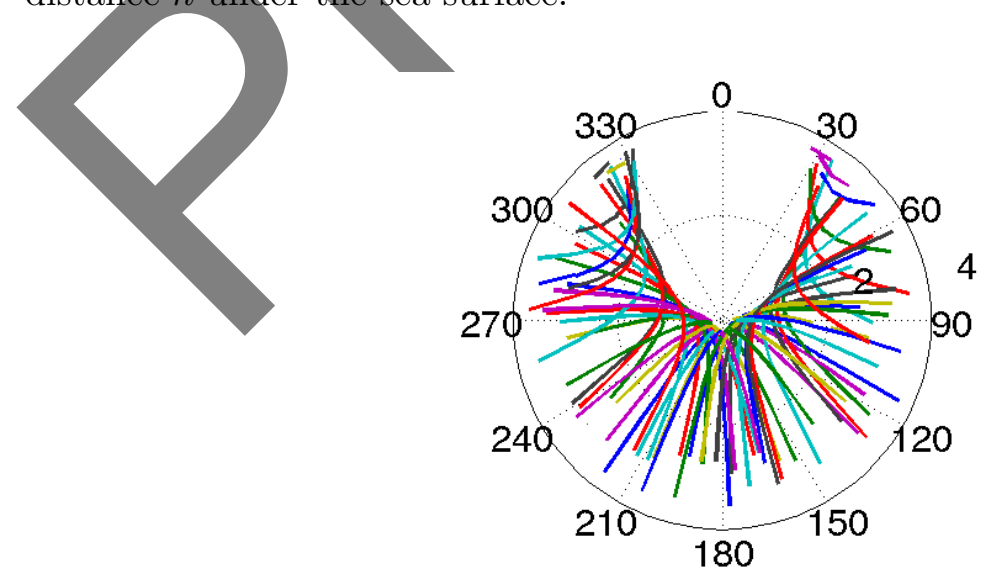

Fig. 2. Simulation of reflection points on the sea surface for different azimuth directions in degrees. The simulation is done for GPS signals during 24 hours with an antenna height of $1 \mathrm{~m}$ over the sea surface and an elevation cut-off of $15^{\circ}$. The outermost circle (solid line) and the inner circle (dotted line) have diameters of $4 \mathrm{~m}$ and $2 \mathrm{~m}$, respectively. 

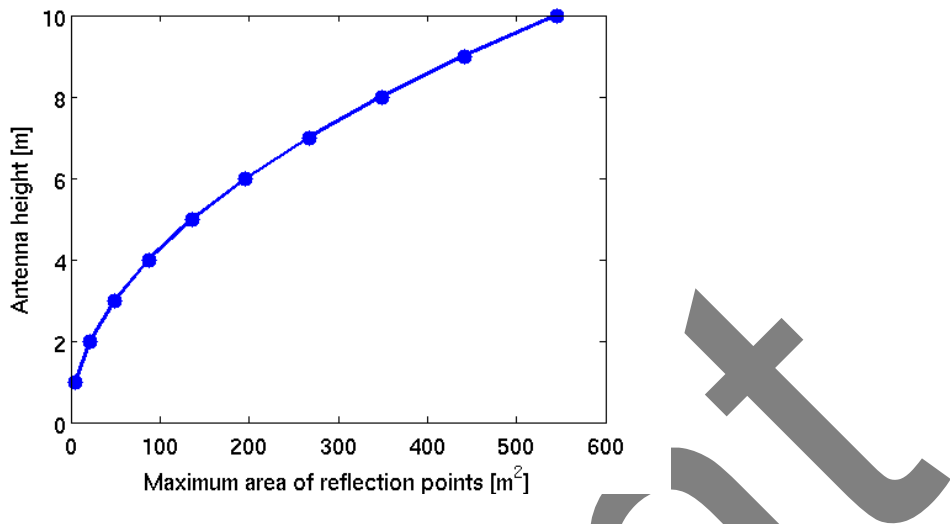

Fig. 3. Simulation of maximum area, defined as a half circle, of the reflection points on the sea surface for different heights of the antenna over the sea surface. The simulation is done for GPS signals during 24 hours with an elevation cut-off of $15^{\circ}$. Additionally, this figure illustrates how the area changes during different stages of a tidal cycle.

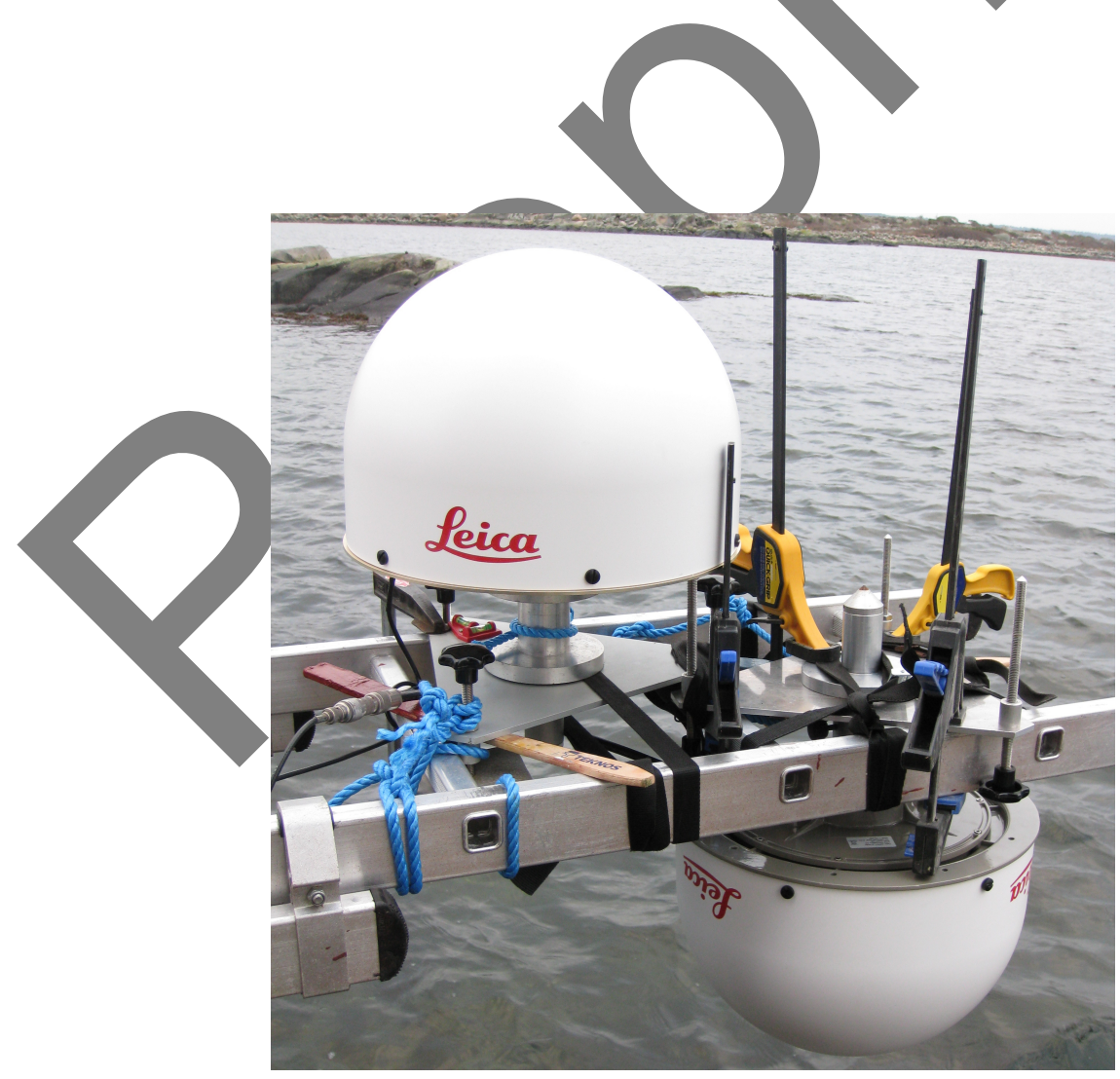

Fig. 4. The experimental setup of the GNSS-based tide gauge at the Onsala Space Observatory. 
a)

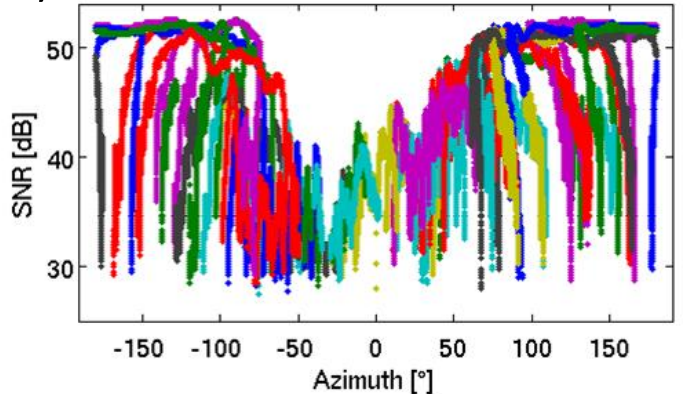

c)

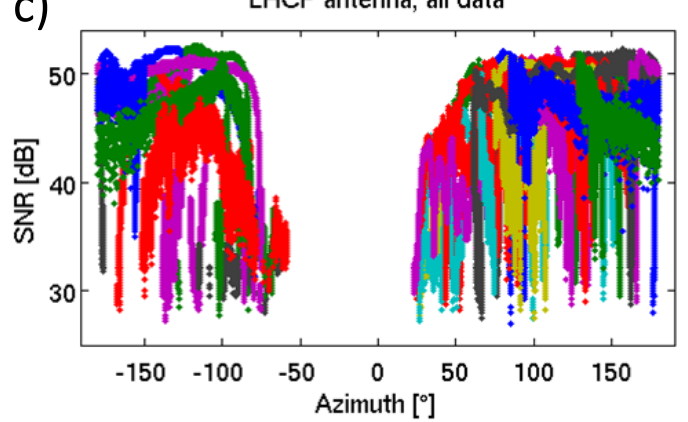

e)

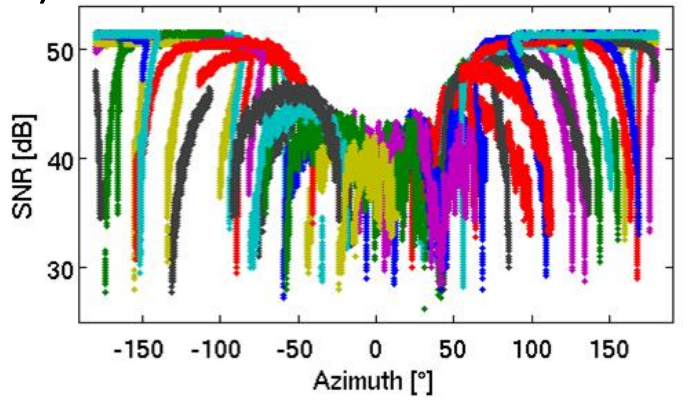

g)

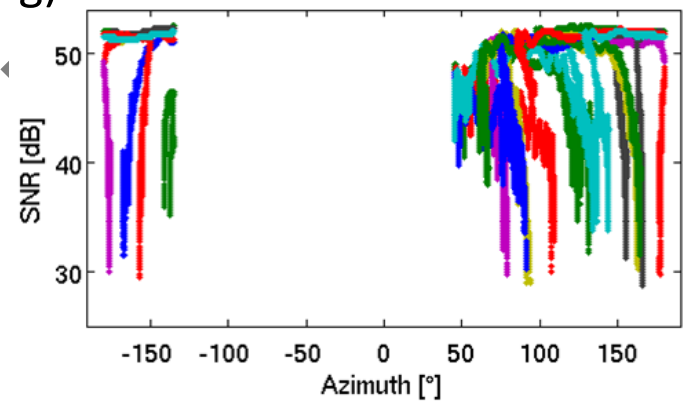

i)

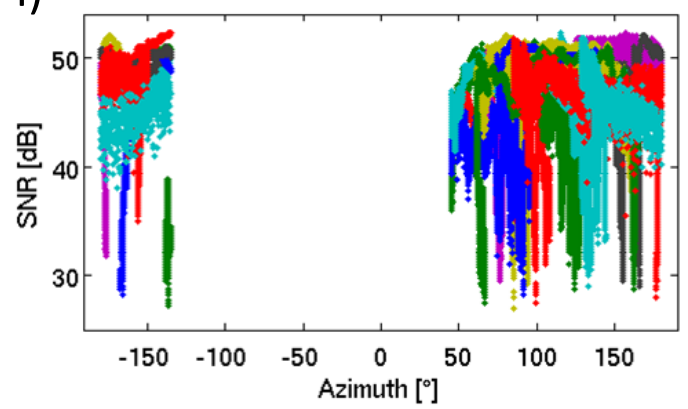

b)

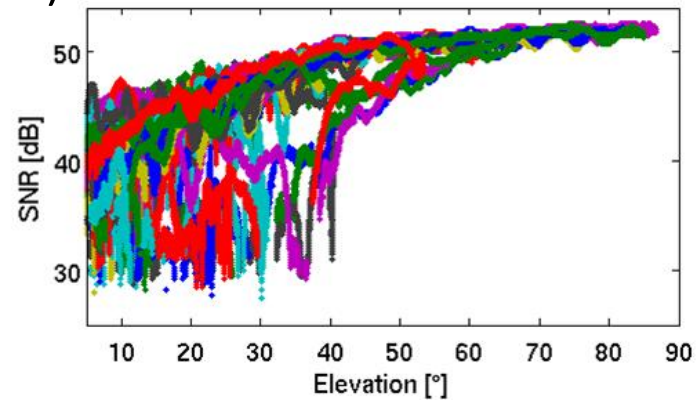

d)

LHCP antenna, all data

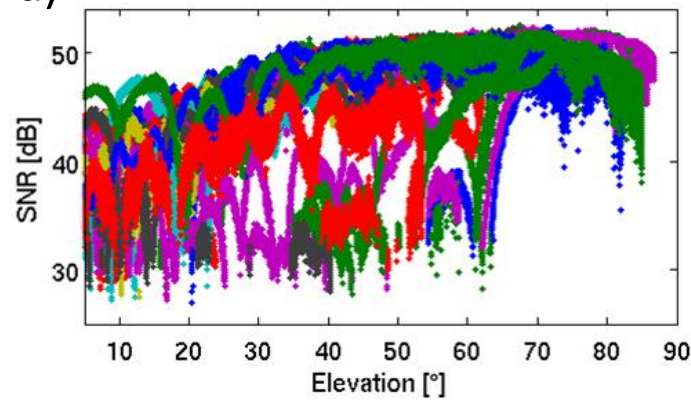

f)

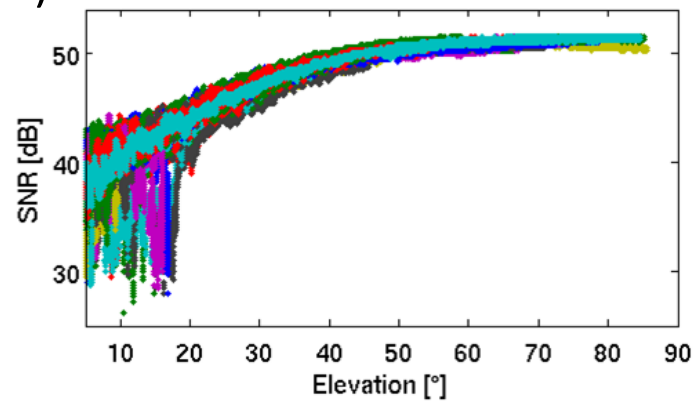

h)

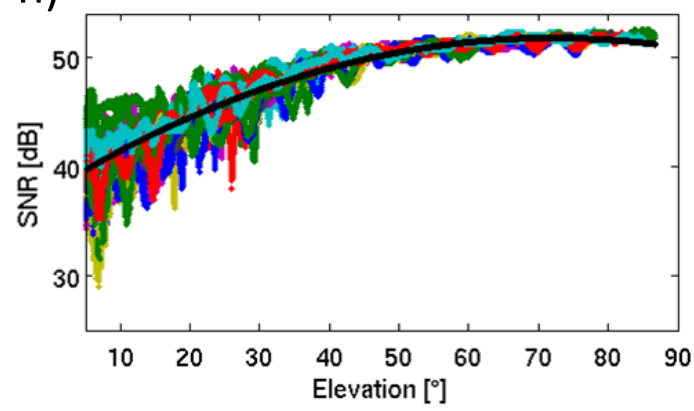

j)

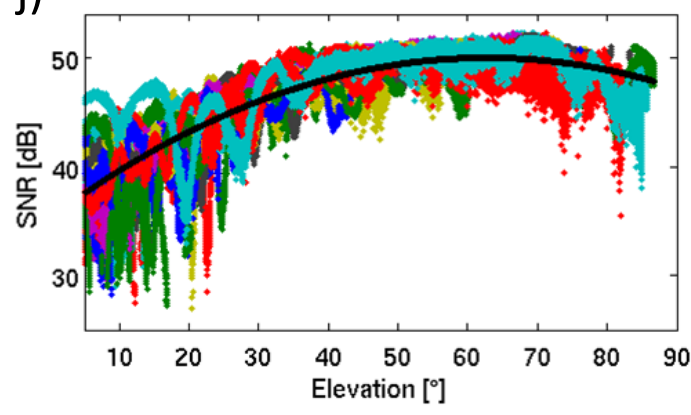

Fig. 5. SNR versus azimuth (left column) and elevation (right column) during 12 hours. Values are presented for all data 1 eived with the RHCP antenna (a and b) and LHCP antenna (c and d). As a comparison, data from the IGS station ONSA are presented for the same time period (e and f). Furthermore, data from after the azimuth mask was applied are presented for the RHCP antenna ( $\mathrm{g}$ and $\mathrm{h}$ ) and for the LHCP antenna ( $h$ and $\mathrm{j}$ ). The polynomial fit to the SNR for each antenna is also shown (h and $\mathrm{j}$ ). 


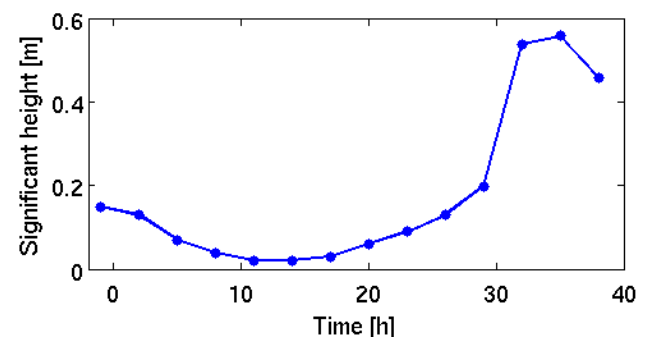

Fig. 6. Significant height of wind waves and swell, in meters, from model data provided by the Swedish Meteorological and Hydrological Institute. The data were produced from the Simulating WAves Nearshore (SWAN) model with forcing from a regional climate model. The location of the grid point is N 57.46 E 11.95 (the GNSS-based tide gauge is located at N 57.39 E 11.92).

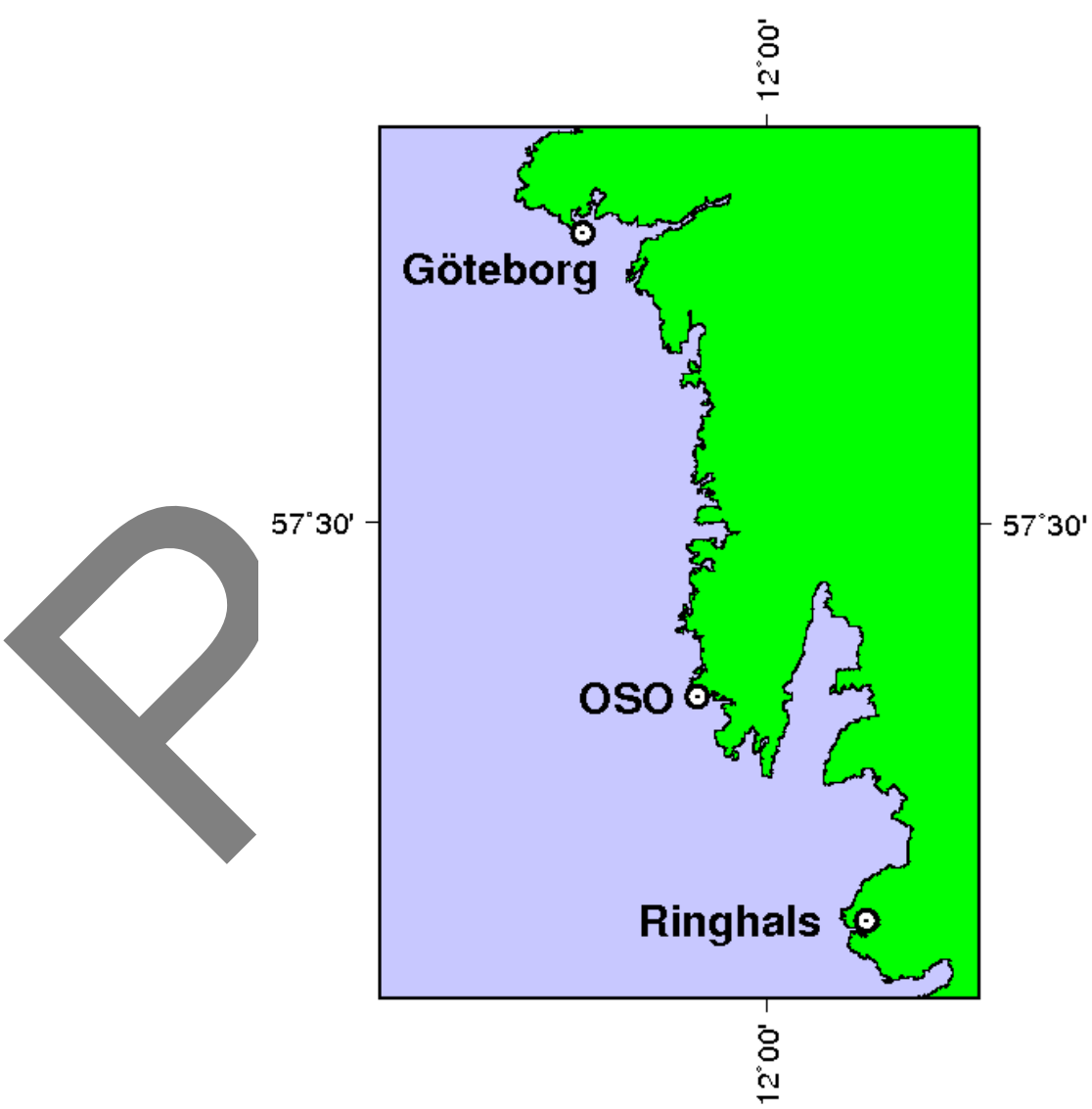

Fig. 7. Map of parts of the Swedish west coast showing the locations of the traditional tide gauges at Göteborg and Ringhals, and the GNSS-based tide gauge at the Onsala Space Observatory (OSO). The distances are about $33 \mathrm{~km}$ between Göteborg and OSO, and $18 \mathrm{~km}$ between OSO and Ringhals. 


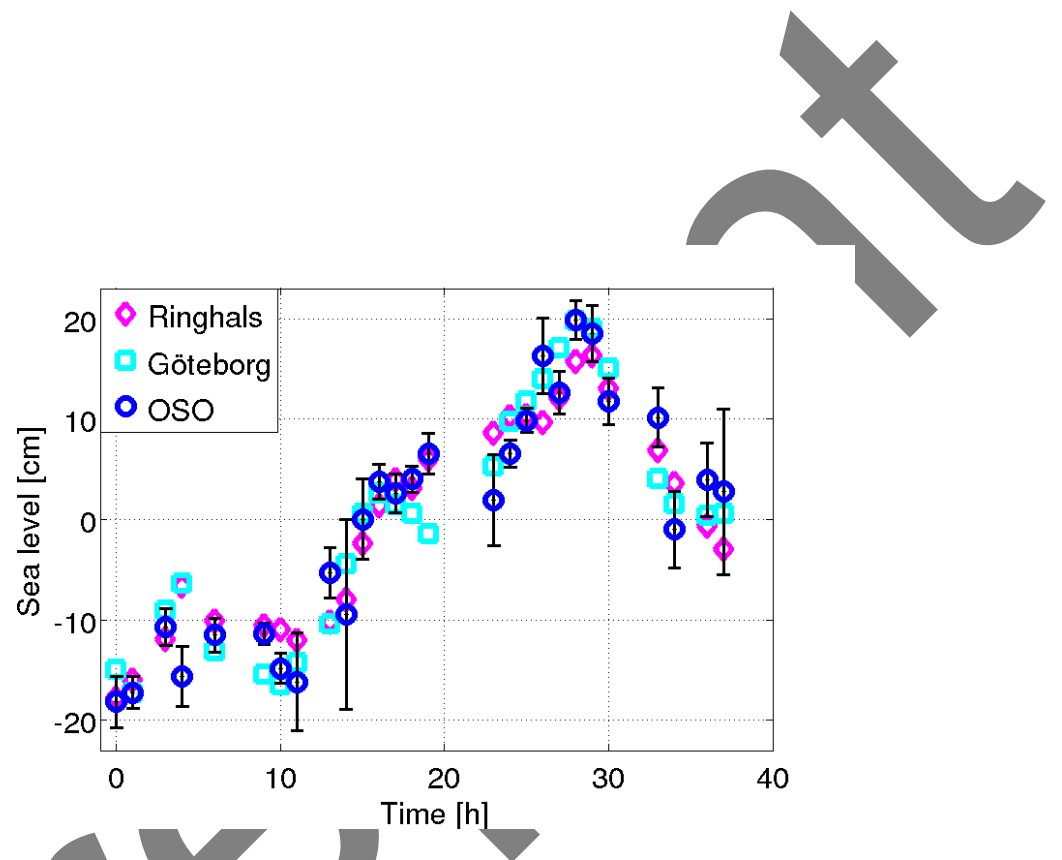

Fig. 8. Time series of sea level derived from the GNSS-based tide gauge (circle) at the Onsala Space Observatory (OSO) and from two stilling well gauges at Ringhals (tilted square) and Göteborg (square) about $18 \mathrm{~km}$ south of and $33 \mathrm{~km}$ north of OSO, respectively. The mean is removed from each time series and the pairwise RMS agreement is better than $4 \mathrm{~cm}$. Error bars for the GNSS-based tide gauge time series consists of standard deviations from each solution multiplied by a factor of 10 . 